Die wirkliche Formel für die Brenzcitronensäure ist demnach $\mathrm{C}^{5} \mathrm{H}^{4} \mathrm{O}^{3}$, und diese nimmt zum $\mathrm{Hydrat} \mathrm{H}^{2} \mathrm{O}$ auf, wie gewöbnlich.

Kann unan nun aus dieser Zusammensetzung Einiges über die Bildung der Brenzcitronensäure schliefsen? Das wäre wohl zu voreilig, so lange ein so wesentliches Product, als die zuvor erwähnte geistige Flüssigkeit noch nicht genau analysirt worden ist. Ich überlasse daher Hrn. Bo ullay die Beendigung dieses Gegenstandes, der gewissermafsen ihm angehört, da er die noch zu untersuchende Substanz cntdeckt hat.

III. Veber die künstliche Aepfelsäure Scheele's; oon Hrn. R. T. Guérin Varry.

(Auszug aus den Ann. de chim. et de phys. T. LII p. 318)

In einer Abhandlung über die Gummi-Arten, von der in dem nächstfolgenden Artikel das Wesentliche mitgetheilt werden wird, glaubte Hr. Guérin bercits an der Eincrleiheit der künstlichen und der natürlichen depfelsäure zweifeln zu dürfen; zur Gewifsheit wurde ihı diefs aber erst durch die Versucbe, deren Beschreibung den Gegenstand des vorliegenden Aufsatzes ausmachen. Das Hauptresultat dieser besteht nun darin, dafs die künstliche Aepfelsäure Sch e ele's eine von der natürlichen verschiedene, eigenthümliche Säure ist, von solcher Zusammensetzung, dafs sie sich als eine Verbindung von Oxalsäure und Wasserstoff betrachten läfst. Er nennt sie daher acide oxalhydrique, was für das Folgende durch Hydroxalsäure (Kleewasserstoffsäure) wieder gegeben seyn mag.

Bereitung der Säure. Man übergiefse 1 Th. Mimosengummi mit 2 Th. einer mit der Hälfte ihres Ge- 
wichts an Wasser verdünnten Salpetersäure in einer Retorte, welche das vierfache Volum des Gemenges falst und mit einer tubulirten Vorlage versehen ist. Man erbitze das Ganze gelinde bis das Gummi gelöst ist, und hebe es beim Erscheincn salpetriger Dämpfe vom Feuer. Nach Auflı̈̈ren der Entwickelung von Salpetergas, die beträchtlich ist, halte man die Flüssigkeit eine Stunde lang in Sieden, verdünne sie mit dem vierfachen Gewichte Wasser, neutralisire sie vollkommen durch Ammoniak, und fälle die Oxalsäure, die sich fast immer in geringer Menge gebildet hat, durch salpetersauren Kalk. Die röthlich gelbe Flüssigkeit filtrire man nun, fălle sie durch essigsaures Blej, bringe den Niederschlag auf ein Filtrum, wasche ihn so lange, bis das Abgelaufene nicht mehr durch Schwefelwasserstoffgas geschwärzt wird, und zersetze ihn darauf durch Schwefelsäure, die mit dem sechsfachen Grewicht an $W$ asser verdünnt ist.

Die so erhaltene Säure ist gelb; man dampfe sie bei gelinder Wärme ein, neutralisire sie, $i$ nach hinreichender Concentration, durch Ammoniak, und fabre mit dem $\mathbf{A b}$ dampfen bis zum beginnenden Anschiefsen fort. Die erhaltenen, schwarz ausschenden Krystalle entfärbe man durch gereinigte Thierkoble, fälle die entfärbte Flüssigkeit durch essigsaures Bleioxyd und behandele den Niederschląg wie zuvor. Die erhaltene Flüssigk eit dampfe man nun in der Wärme bis zur Syrupsconsistenz, und dann, unter der Luftpunpe, bis zur Trockne. Die Abdampfung darf nicht zu weit getrieben werden, denn es tritt ein Punkt ein, wo sie gelb und zersetzt wird.

In diesem Zustand enthält die Säure auf zwei Atome ein Atom Wasser. 1000 Th. Arabin *) mit 2000 'Th. Salpetersäure behandelt, geben im Maximo 2,8 Th. $\mathrm{Hy}$ droxalsäure. 1000 Th. Zucker mit dem gleichen Gewichte Salpetersäure liefern im Maximo 3,5 Th., Stärkmehl dagegen $3,1 \mathrm{~Tb}$. einer der Hydroxalsäure vollkommen glei-

-) Man sehe den folgenden Aufsatz. 
chen Säure. Wahrscheinlich ist es auch diese Säure, welche man nach Thénard und Berzelius erhält, wenn Traubenzucker, Mannazucker, Schwammzucker, Pollenin, fette Oele etc. mit Salpetersäure behandelt werden. Die besondere Säure, welche man nach Berzelius bekommt, wenn man Salpetersäure bei gewöhnlicher Temperatur auf Zucker einwirken lälst, konnte Hr. G. nicht erhalten. Er liefs $1 \mathrm{Th}$. Zucker mit $1 \mathrm{Th}$. rauchender durch ein gleiches Gewicht Wasser verdünnter Salpetersäure stehen, und digerirte einmal vier Tage lang und ein anderes Mal einen Monat hindurch, konnte aber keine Spur von jener eigentbümlichen Säure auffinden, nur war die Lösung gelblich.

Eigenschaften der Hydroxalsäure. Sie hat die Consistenz eines dicken Syrups, bei $20^{\circ} \mathrm{C}$. die Dichte 1,416, ist farb- und geruchlos, schmeckt sehr wie Oxalsaiure, ist an der Luft sehr zerfliefslich, und kommt dadurch auf die Dichte 1,375 herab, siedet bei $105^{\circ} \mathrm{C}$., löst sich in Wasser und Alkohol in allen Verbältnissen, sehr wenig in Aether, selbst siedendem, auch in Terpenthinol selbst beim Sieden nur höchst unbedeutend. Mit trocknem Bleioxyd erhitzt, dieses sey in Ueberschufs oder gerade zur Sättigung hinreichend, verliert sie immer gleichviel Wasser.

Diese wasserhaltige Säure, in einer Flasche mit eingeriebenem Stöpsel einen Monat stehen gelassen, setzte Krystalle ab, die, hinsichtlich der Form, der Oxalsäure ähnlich waren, in ibren Eigenschaften aber von dieser gänzlich abwichen. Wahrscheinlich waren sie Hydroxalsäure, da sie alle Eigenschaften derselben besafsen.

Die wasserhaltige Hydroxalsäure bildet mit Salzbasen vollkommen charakterisirte Salze. Sie giebt mit Kalk-, Strontian - und Barytwasser Niederscbläge, die sich im geringen Ueberschufs der Säure wieder lösen. Sie ähnelt darin der Weinsäure, unterscheidet sich aber von dieser 
dadurch, dafs sie concentrirte Lösungen von Kali oder Kalisalzèn nicht fällt. Mit der natürlichen Aeprelsäure kann sie auch nicht verwechselt werden, da diese die genannten drei Alkalien nicht fält.

Salpetersaures Silberoxyd, salpetersaures, basisches und neutrales essigsaures Bleioxyd werden von der $\mathrm{Hy}$ droxalsäure weifs und voluminös gefallt. Sie löst Zink und Eisen unter $W$ asserstoffentwickelung auf, greift aber Zinn weder kalt noch warm an.

Die an feuchter Luft entstandene Lösung der Säure veränderte sich, bei $18^{\circ}$ bis $25^{\circ}$, in Laufe dreier Monate nicht; eine verdünntere Lösung bedeckte sie jedoch nach einigen Tagen mit Schinmel.

$1 \mathrm{Th}$. Hydroxalsäure mit $3 \mathrm{Th}$. Salpetersäure in einer bis auf eine sehr kleine Oeffnung verschlossene Flasche eiuen Monat lang, in gewöhnlicher Temperatur, unter täglichem Umschütteln stehen gelassen, hatte nach $\mathrm{Ab}$ lauf dieser Zeit, unter Entwickelung von Solpetergas und Kohlensäure, viele gut krystallisirte Oxalsäure abgesetzt Erwärmte man das Gemenge bis zum schwachen Sieden, so fand dieselbe Reaction (wahrscheinlich nur schneller) statt.

Mit 1 Th. concentrirter, durch ein gleiches Gewicht an Wasser verdünnter Schwefelsäure erhitzt, giebt sie Kohlensäure und schweflige Säure. Mit Schwefelsäure und Manganbyperoxyd in einem Destillationsgefarfs gelinde erwärmt, geht die Hydroxalsäure in Ameisensäure über, erkennbar durch ihre reducirende Wirkung auf Quecksilberoxyd, salpetersaures Quecksilberoxyd und salpetersaures Silberoxyd.

Chlorwasserstoffsäure hat in der Kälte keine Einwirkung auf die Hydroxalsäure; in der Wärme wird die Flüssigkeit gelb, ohne dafs sich Gas entwickelt.

Bis $106^{\circ}$ C. erwärmt, erleidet dic Säure eine Veränderung. Im Destillationsgefälse erhitzt, giebt sie unter 
Aufschwcllen die-gewöhnlichen Producte stickstoffreier organischer Substanzen, während eine voluminöse, schwicrig einzuäschernde Kohle zurückbleibt.

Zusammensetzung. Durch Zcrlegung des Bleisalzes und eines Zinksalzes erhielt Hr. G. folgendes Resultat *):

\begin{tabular}{lcc|c|c} 
& \multicolumn{2}{c|}{ Bleisalz. } & Zinksalz. & \\
& Beobachtet. & Berechnet. & Beobachtet. & Atome. \\
Sauerstoff & $\mathbf{6 4 , 5 7}$ & $\mathbf{6 3 , 6 2}$ & $\mathbf{6 3 , 2 1}$ & $\mathbf{6}$ \\
Kohlenstoff & $\mathbf{3 1 , 3 5}$ & $\mathbf{3 2 , 4 2}$ & $\mathbf{3 3 , 1 4}$ & $\mathbf{4}$ \\
Wasserstoff & $\mathbf{4 , 0 5}$ & $\mathbf{3 , 9 6}$ & $\mathbf{3 , 6 5}$ & $\mathbf{6}$ \\
\cline { 2 - 3 } & $\mathbf{1 0 0 , 0 0}$ & $\mathbf{1 0 0 , 0 0}$ & $\mathbf{1 0 0 , 0 0}$ &
\end{tabular}

was also sehr von dem Resultat der Liebig'schen Analyse der natürlichen Aepfelsät.re abweicht **). Dic Schleimsäure enthält etwas weniger Sauerstoff als die Hydroxalsïure; ihre Bildung muls also die der letzteren bei Behandlung des Gummi's mit Salpetersäure kurze Zeit rorangehen.

100 Th. Hydroxalsaiture sättigen eine Oxydmenge, die 10,603 Sauerstoff, d. h. ein Sechstel so viel als die Säture enthält.

Wasserfrei konnte Hr. G. die Hydroxalsäure nicht erhalten. Die wasserhaltige enthält 5,65 Procent Wasser, besteht demnach aus 2 Atomen wasserfreier Säure und 1 Atom Wasser.

Salze. Mit Ammoniak giebt die Hydroxalsäure zwei Salze, ein neutrales, das nicht krystallisirt, und ein saures. Letzteres ist das bereits zuvor erwähnte, und krystallisirt in vierseitigen zugeschärften Prismen, ist farblos und an der Luft unveränderlich, schmeckt schwach sauer, löst sich in Wasser bei $15^{\circ}$ C. zu 1,22 Procent, und bei

-) Das Detail dieser und aller folgenden Analysen ist nicht mitgetheilt. Ihre Richtigkeit wird also nur durch das Zutranen verbürgt, welches man Hrn. G. schenken will.

$P$.

-) Selbst von dem der neuesten (Annal. Bd. XXVII S. 195), nach welcher diese Säure isomer ist mit der Citronensäurc. $P$. 
bei $100^{\circ}$ zu 24,35 Procent, ist unlöslich im kalten und löslich im siedenden Alkohol, fängt an sich bei $110^{\circ} \mathrm{C}$. zu zersetzen; seine Lösung giebt, mit Ammoniak neutralisirt; beim Verdampfen an der Luft wieder Krystalle vom sauren Salz. Es besteht nach Hrn. Gr. aus 89,49 Proc. oder 2 Atomen Säure, 5,14 Proc. oder 1 Atom Ammoniak und 5,37 Proc. oder 1 Atom Wasser.

Mit dem Kali giebt sie gleichfalls ein saures und cin neutrales Salz. Erstes wird durch unvollkommene Sättigung der Säure mit doppelt-kohlensaurem Kali erbalten, und schiefst in durchsichtigen nadelförmigen Prismen mit schicfer Basis an; letzteres, nach-vollkommener Sättigung mit jenem Bicarbonat entstehend, bildet durchsichtige schicfe Prismen mit rhomboïdaler Basis.

Von den Natronsalzen ist weder das neutrale noch das saure krystallisirbar.

Neutrales Barylsalz fallt in weifsen Flocken nieder, wenn das neutrale Ammoniaksalz mit. Chlorbarium in concentrirter Lösung vermischt wird; es ist in viclem kalten Wasser löslich, und schiefst, beim Abdampfen, nur in Plättchen an. Ein saures Salz, das bei freiwilliger Eindunstung einen gummiähnlichen Rückstand hinterläst, entsteht durch Anwendung der Säure in Ueberschufs.

Neutrales Strontionsalz bereitet und verbailt sich wie das neutrale Barytsalz; das saure krystallisirt dagegen in Prismen. Das neutrale Kalksalz ist, selbst warm, sebr wenig löslich und unkrystallisirbar, das saure krystallisirt in 4seitigen Prismen.

Das Zink giebt ein Sesquisalz, bestehend aus 68,98 Proc. oder 3 At. Sïure, 24,64 Proc. oder 2"At. Zinkoxyd und 6,38 Proc. oder.2 At. Wasser. Es ist in kaltem Wasser gar nicht, in siedendem sehr wenig löslich, löslich aber in seiner eigenen Säure. Seine Lösung röthet Lackmus. Es wird bereitet durch Auflösung von gekörntem Zink in verdünnter Hydroxalsäure, wobei es unter Wasserstoffentwickelung als weifses Pulver niederfällt. 
Das Bleisalz besteht aus 40,34 Proc. oder 1 Atom Siuure und 59,66 Proc. oder 1 At. Oxyd, enthält kein Krystallwasser, löst sich in kaltem Wasser gar nicht, in siedendem sehr wenig und fällt daraus beim Erkalten in Blättchen wieder heraus, ist unlöslich in überschüssiger Säure und in Alkohol, fängt bei $120^{\circ} \mathrm{C}$. an gelb zu werden, schmilzt bei $135^{\circ}$ zu gelben Tropfen, die bei $140^{\circ}$ roth und bei $150^{\circ}$ zerselzt werden, giebt, in einer Röhre verkoblt, einen Rückstand, der, noch heifs in die Luft geschültet, glühende Funken mit nachziehendem weifsen Rauche bildet, entflammt sich bei Erhitzung mit Salpetersäure wie Pulver, entwickelt mit concentrirter Schwefelsäure anfangs einen dem Weinöl ähnlichen Geruch und später schweflige Säure.

Eisen wird unter Wasserstoff-Entwickelung von der Hydroxalsäure gelöst, und die Lösung hinterläfst eingedampft einen gummigen Rückstand.

Kupfer, und noch besser Kupferoxyd lösen sich in ihr, aber das bläuliche Salz krystallisirt nicht.

Quecksilberoxyd giebt ein weifses, fast unlösliches Salz, das Lackmus röthet.

Chromoxydul liefert ein sauer reagirendes Salz in durchsichtigen, farblosen, schiefbasigen Prismen.

\section{Ueber die Gummiarten;}

von Hrn. R, T. Gućrin.

(Auszug aus den Ann. de chim. et de phys. T. XLIX p. 249.)

$\mathbf{U}$ nter dem Namen Gummi versteht der Verfasser alle die Pflanzenstoffe,' welche sich durch Salpetersäure in Schleimsäure umwandeln lassen, ohne dabei krystallisirbar zu seyn, wie der Milchzucker, dem sonst auch die nämliche Eigenschaft zukommt *). Hauptsächlich sind es

-) Um das Schwankende in der bisherigen Begriffsbestimmung vom Gurnmi fühlbar zu nachen, bemerkt der Verfasser, dafs Hr. Ro- 